\title{
METHODS OF STABILITY ANALYSIS IN NONLINEAR MECHANICS*
}

\author{
R. L. Warnork and R. D. Ruth \\ Stanford Linew Accelerator Center \\ Stanford University, Stanford, California 94309 \\ W. Cabella' \\ Department of Physics \\ University of Colorado \\ Boulder, Colorado 89309 \\ K. Ecklund \\ Department of Physics \\ Princeton University \\ Princeton, New Jersey 08544
}

\section{January 1989}

\begin{abstract}
We review our recent work on methods to study stability in nonlinear mechanics, especially for the problems of particle accelerators, and compare our ideas to thoce of other authors. We emphasize methods that (a) show promise as practical design tools, (b) are effective when the nonlinearity is large, and (c) have a strong theoretical basis.
\end{abstract}

\section{TABLE OF CONTENTS}

1. Introduction

2. Tracking

3. Perturbation Theory

4. Hamillon-Jacobi Method

5. Cenonical Transformations and Bouncis on the Motion for Time $T$

6. Pull Turn Maps

7. Functional Equation for Invariant Surfaces

8. Conclusions

- Wort espported by the Department of Energy, contratls DE-AC03-765F00515 aNib DE-FG02-86ER40302.

t Curnent addrew: Stanford Linear Aceelrator Center, Stanford Univenity, Stanford, Californis 94309.

Contributed to the Proceedings of the U.S. Particje Accelerator School, 


\section{CONF Y40?208-.4}

\section{INTRODUCTION}

Emerging problems of noulinear mechanics in accelerators are taking on a new order of difliculty. Low emittance damping rings and synchrotran light sourcess have strongly nonlinear lattices and complications dur to wigglers and undulators. l.arge hadron rings are hard to analyze because of their sheer size and the presrnter of high multipoles, random and systenatic, in superconducting inagnets. Largeaperture antiproton collectors and conventional-magnet boosters for hadion col. liders have pronsinent nonlinear effects. As the problems become more diffirult. the shortcomings of traditional analysis become morc apparent.

The traditional methods are based on tracking of single particles and perturbation theory. We first comment of efforts to extend and improve these establislied methods.

\section{TRACKING}

Tracking is the only method that is generally applicable, in that it gives results in any region of phase space. Its drawbacks are (i) computational expense, and (ii) the difficulty of interpreting the results.

To minimize expense while maintaining accuracy, it is important that the method used to integrate Hamilton's equations generate a symplectic time evolution map. (This is truc at least for circular machines; for single-pass beam transport the symplectic condition is less important.) Thin-element "kick codes" generale symplectic time evolutions. By putting together sufficiently many thinelement kicks, one can approximate the effect of a thick element to any desired accuracy. There exist more sophisticaled symplextic integrators, ${ }^{1,2,3,4}$ analogous to standard numerical integralion schemes foe general differential equations. Ruth's fourth-order explicit integrator ${ }^{5}$, ten tested with good results in a skeleton tracking code. A ge . al purpose tracking code using a high order explicit symplectir integrator remains to be written. Also, the "numeri:al analysis" of

.) jilet tic integrators should be developed; i.e., the various algorithms should be compared through rigorous error analysis and experimental tests, as in the estahlished theory of numerical integration of differential equations. We hope that th:subject of symplectic integrators will thrive, sirse we see an important role for in the general approach to stability questions outlined below. Fortunately, there increasing interest on the part of mathematicians and physicists in various fields.

In another approach to reduction of rost, one tries to construct an explirit map to describe propagation through a segment of an atcelerator, the segment being anything from a single lattice element to the whole lattice. We consider this idea in Section 6.

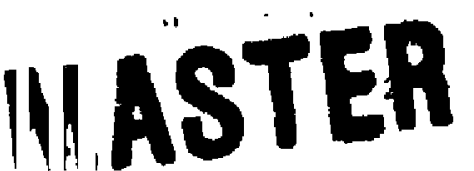


The problem of how to interpret tracking data is sometimes easy ant some times dificult. If the computed trajectory leaves the desired pliysical apert ure. w. have the obvious conclusion that the motion is effectively unstable. To srrify ilue conclusion, we need only provide a proof that there is no problem of mumarm al error, which often can be done hy backtracking to the initial conditions. On the other hand, if the trajectory stays within the required aperture for many thom sands of turns, we do not know what to conclude, since the number of possible. turns in a computation is always very small compared to the number achimvinl by a real heau. In fact, there are examples of tracking runs in which the artsit appeared to be stable for $10^{5}$ turns or so, and thell suddenly became unst itble.

In view of this ambiguity, it is interesting to examine tracking data in wass that go beyond the simple requirement of confinenent to a defined aperture. One possiblity is to judge che data for degree of deviation from linear motion. The degrey of deviation, which nuy be defined in several similar ways, is called the "smear"? Through experience and intuition one tries to set a safe limit for the snear. In Ref. 8 we gave an example, a two-resonance model, in which the smear was quite small even though the system was near the onset of large-scale chaotic motion. This and other considerations suggest that a rule-of-thumb for maximum allowed smear is not likely to be universal. A correct rule wipht depend strongly on the tune and be different for difierent kinds of lattices. Neves't iess, optimizing the lattice to reduce the smear is clearly a worthwhile ain.

An idea with a stronger motivation is to test for confinement of the orbit to an invariant surface. On an invariant surface the action $I$ is a function of the angle variable and the orbital position s which may be written as

$$
\mathrm{I}=\mathrm{J}+G_{\boldsymbol{*}}(\mathrm{J}, \mathbf{3}, \mathbf{s}) \text {. }
$$

Here $J$ is the invariant action, and $G$ is the Hamilton-Jacobi generating function which solves the Hamilion.Jacobi equation,

$$
H\left(\mathrm{~J}+G_{4}, \mathbf{s}, s\right)+G_{2}=H^{(1)}(\mathrm{J})
$$

The solution $G$ generates , canonical transformation $(I, \Phi) \rightarrow(J, \%)$ which is defined by (2.1) together with the following equation:

$$
y=5+G_{\mathrm{J}}(\mathbf{J}, \mathbf{s}, \mathrm{s})
$$

Bold-faced quantities are vectors with dimension equal to the number of degrews of freedom, and subscripts denote partial derivatives. The generator $G$ is periodir in 5 with period $2 \pi$, and periodic in $s$ with period $C$. 


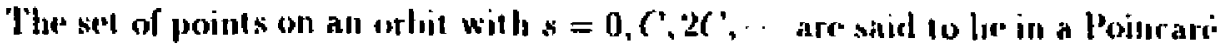
surface of section. If lhe molion is regular and num.resonant, those points lie on

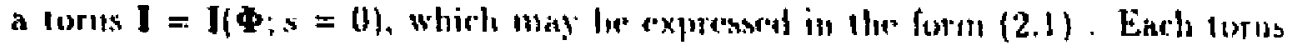
is latheled by a value of the invariant $\mathbf{J}$, which is the average id $\mathbf{I}(\boldsymbol{\Phi} ; \mathbf{s}=\mathbf{0}$ ) oved D. We can approximble the torus, When it exists, lyy fittug tracking dala to it smooth surface. For instance, for betatron motwen in two degrees of freedom we can determine two smooth. I wo dimemsional surfaces lo pass through the points

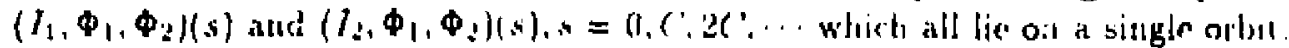
Since the angle coordinates $\left(\Phi_{1}, \Phi_{2}\right)$ do not he on a regular grid, one must have a surface fitting program which can handle scattered data. Wh have used the IMSSL program IQHSCV, which produces a piece-wise polynomial surface whicl passess through the given point? and has rontinuous first derivatives.

In Figures 1 and 2 we show results for the North Dampiug Ring of the SI.(". Normalizing to the invariants, we plot $l_{1} / J_{1}$ and $l_{2} / J_{2}$ versus $\left(\Phi_{1}, \Phi_{2}\right)$, with the origin al zero. Each of the surfaces was fitted to $\mathbf{8 0 0 0}$ points obtained from $\mathbf{8 0 0 0}$ turns of a fourth order symplertic tracking code. Linear motion would correspond to flat surfaces, $I_{1} / J_{1}=1$. The inilial displacements of the orbit correspont to sbout $3.7 \mathrm{~mm}$ (horizontal) and $2.6 \mathrm{~mm}$ (vertical) it the septum magnet. Theste are large but sustainable injection offsets for the ring considered. The smear is inpressively large.

To gain more insight it is useful to compute the two-dimensional Fourier trans. form of $\mathrm{I}\left(\boldsymbol{\Phi}_{;} s=0\right)$, which gives us the Fourier coefficients of $G_{\star}$ :

$$
I=J+\sum_{m} i m g_{m}(0) e^{2 m}
$$

It is to be emphasized that we are making a Fourier analysis of the invariant surface, not an analysis of the time series $\mathbf{I}(s), s=0, C, 2 C, \cdots$. Although the latter is also of somr interest, in that it can be compared to experiment, it is relatively difficult to interpret. The coefficients $\left.g_{m}(s)\right|_{s=0}$ of $(2.4)$ are the familiar Fourier coefficients of the generating function, which may also be computed in perturbation theory or by direct solution of the Hamilton-Jacol: equation. If $\nu=\left(\nu_{1}, \nu_{2}\right)$ is the tune and $m \cdot \nu$ is close to an integer, the consequent resonance shows up as a relatively large value of $\mathrm{m} g_{m}$ in (2.4), since, as is well known, the latter is amplified by a small divisor $\sin (\pi \mathrm{m} \cdot v)$. Thus, $(2.4)$ analyzes the motion according to nonlinear resouances in a most direct und elernentary way.

Taking an FFT of the surface in Fig.1 or Fig.2, and retaining modes for $\left|m_{1}\right|,\left|m_{2}\right| \leq 15$, we obtain a Fourier series represuntation of the surface which agres well witl the original (pierewise-polynomial) surface computed by IQII. SCV, and also agrees well with tracking beyond the original 8000 turns. Defining an appropriate metric $\Delta /$ for the difference between the tracked orbit and the 


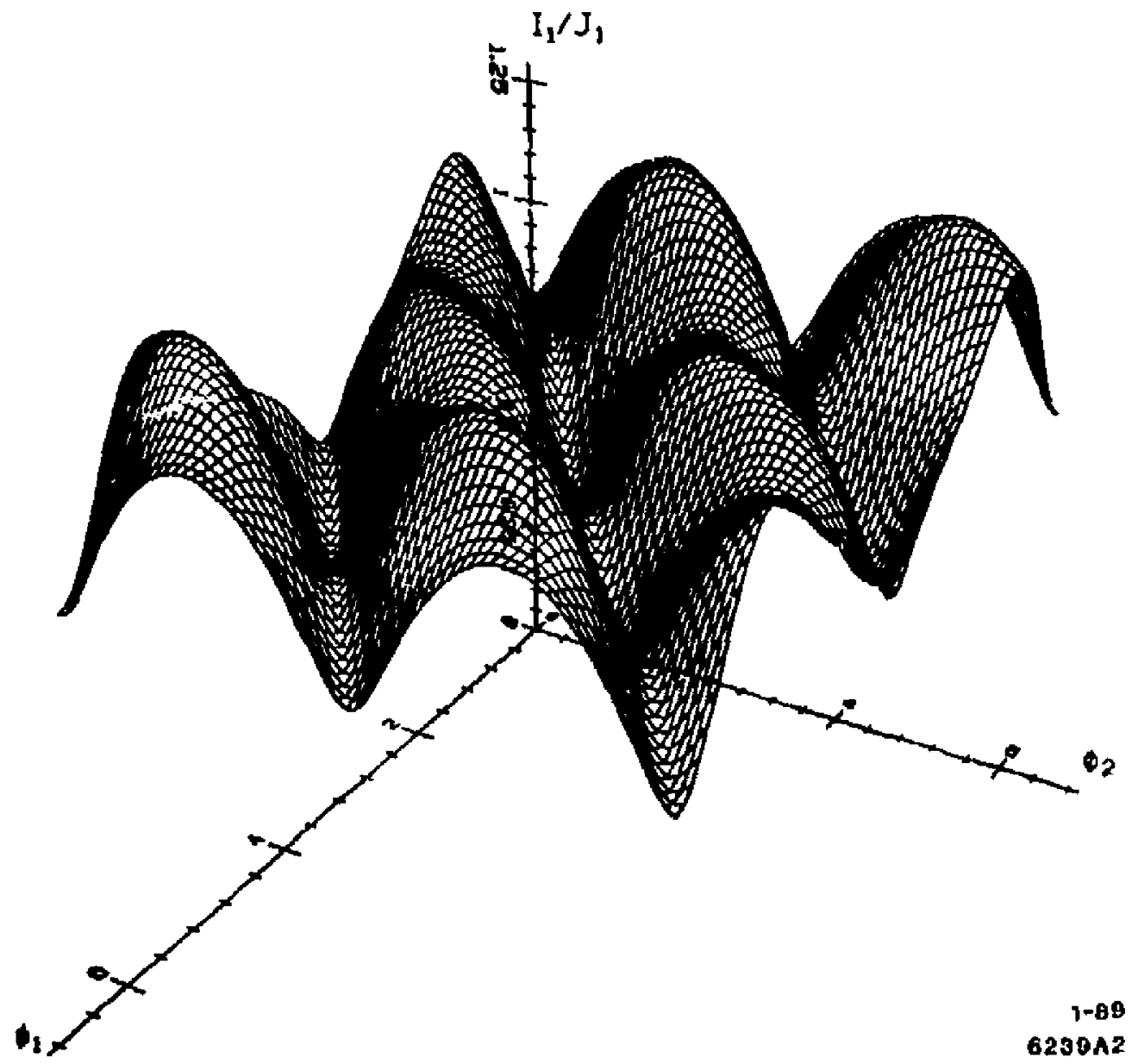

Figure 1 : Invariant surface for SLC: North Damping Iting, obtained by a fit to tracking data. $J_{1}\left(\Phi_{1}, \Phi_{2}\right) / J_{1}$ plotled as function of $\Phi_{1}, \Phi_{2}$. Invariant action $J_{1}=2.489 \cdot 10^{-6} \mathrm{~m}$ 


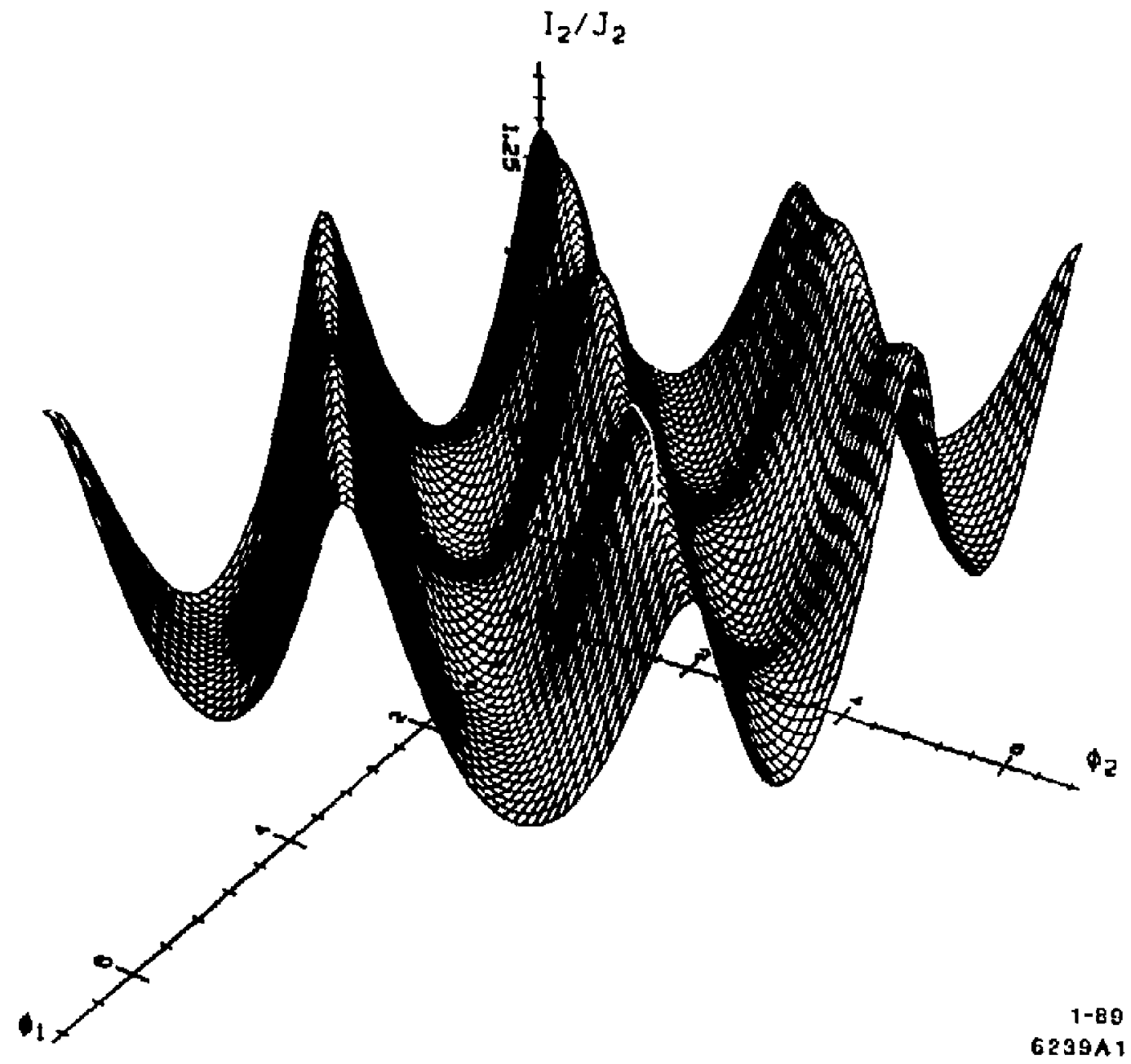

Figure 2 : Invariaut surfncu for SIC: North Damping Ring, obtained by a fit to tracking data. $l_{2}\left(\Phi_{1}, \Phi_{2} j / J_{2}\right.$ plotted as function of $\Phi_{1}, \Phi_{2}$. Invariant action $J_{2}=1.508 \cdot 10^{-6} \mathrm{~m}$ 
Fonerier series (2.4) (the sum of the deviatiens divided by the mumber of orlot

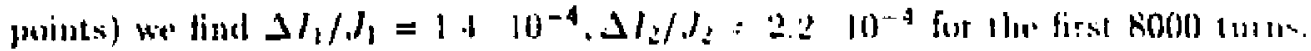

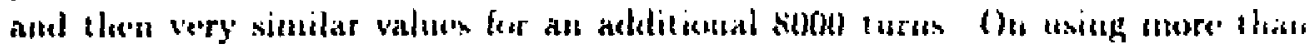

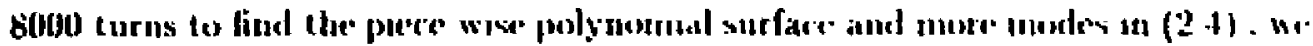

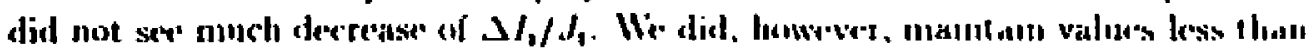

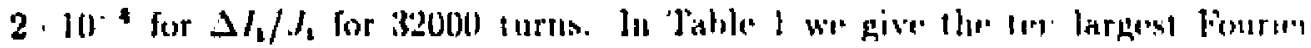
conflicients of $f_{1}$, divided by $f_{1}$.

Table I: Fourier Amplitudes of $\left(_{\phi_{1}}\right.$

\begin{tabular}{|c|c|c|c|}
\hline $\mathbf{m}_{\mathbf{1}}$ & $\mathbf{m}_{2}$ & $\operatorname{He}\left(\mathrm{im}_{1} \mathrm{gm}_{\mathrm{m}}\right)$ & $\ln (i m, g m)$ \\
\hline 2 & .2 & $-2.06 \cdot 10^{-7}$ & $-9.5 \cdot 4 \cdot 10^{-9}$ \\
\hline 1 & 0 & $6.76 \cdot 10^{-4}$ &.$-1.91 \cdot 10^{-9}$ \\
\hline 3 & $\mathbf{0}$ & $\cdot 6.61 \cdot 10^{-8}$ & $2.40 \cdot 10^{-10}$ \\
\hline 4 & .4 & $\cdot 4.12 \cdot 10^{-6}$ & $.3 .77 \cdot 10^{-y}$ \\
\hline 2 & 2 & $1.21 \cdot 10^{-8}$ & $.9 .57 \cdot 10^{-11}$ \\
\hline $\mathbf{5}$ & -2 & $1.17 \cdot 10^{-8}$ & $5.09 \cdot 10^{-10}$ \\
\hline $\mathbf{0}$ & -6 & $\cdot 1.16 \cdot 10^{-8}$ & $-1.59 \cdot 10^{-9}$ \\
\hline 1 & 2 & $-9.34 \cdot 1 u^{-9}$ & $4.48-10^{-10}$ \\
\hline 8 & -8 & $.4 .15 \cdot 10^{-0}$ & $-7.64 \cdot 10^{-10}$ \\
\hline 7 & .4 & $2.98 \cdot 10^{-9}$ & $2.62 \cdot 10^{-10}$ \\
\hline
\end{tabular}

Thus, we have a surface in the form (2.4) which appears to approximate an invariant surface. We comment presently on meass to improse the approximation. but first renark that we have already obtained very reliable information on the important resonances, by means of a modest and convenient romputation. Suct information might be used to optimize the tattice. For instance, one $n$ " ty 10 adjust sextupoles so as to ninimize a "fignre of oemerit" such as thr. "gg.

$$
\sum_{\cdot} \frac{1}{J_{1}} \sum_{\mathbf{m}}\left|m_{\imath} g_{\mathrm{m}}(0)\right|
$$

The approximale invariant surface in the form (2.4) for $s=0$ can be extende: to all s by integration of the Hamilton-Jacobi equation. One then has a generalor which can be refined in accuracy through further iterations of the Hamilion.Jacoln 


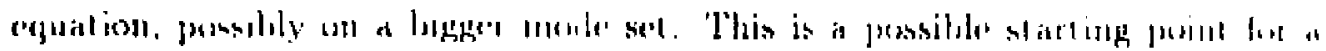

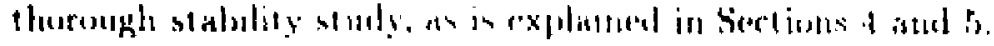

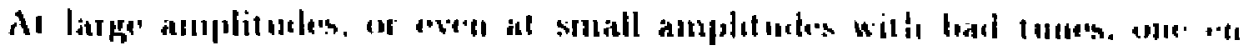

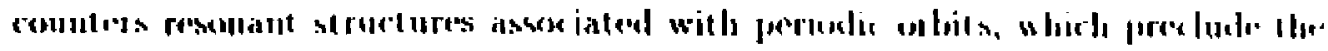

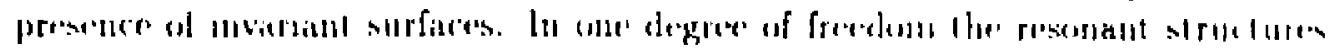

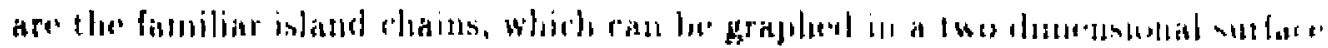

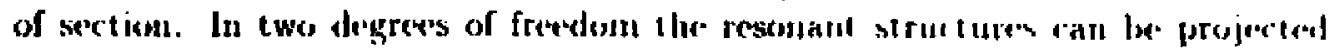
onto a seretion in which s and one of the angles, say $\phi_{1}$, are constant. In Irack itig through a linte number of tums we get essentially zoro population of such

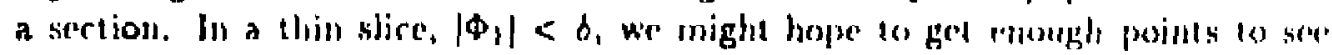
a structure (slightly snetared out), provided that w' can elu very long tracking runs. This might be an interesting application of full-turm maps, which have the potential of greatly reducing computation times; see Section 6 .

\section{PERTURBATION THEORY}

Perturbation theory ${ }^{9}$ is used prinarily to find approximations to invariant surfaces and related quantities such as tune shifts, although it may be used as well to discuss time dependence, ${ }^{10} \mathrm{It}$ is an excellent method for situations in which nonlinear effects are weak. Those situations may be defined as cases in which the frst term of the perturbation series, or at most the first and second terms, provide a good approximation. Unfortunately, there is no such casc in the region of phase space near the dynamic aperture of an accelerator. In the example of the previous section low order perturbation theory would fail drastically, as can be seen immedialely from the rich mode spectrum of Table 1 . There are many appreciattle modes that simply do not appear in low orders.

There have been attempts to estimate the dyamic aperture using perturba. tion theory and certain recipes, but the recipes lack theoretical foundation and inay toe urureliable.

One might bope to save the day by going to higher ord'rs in the perturbation series, but in our view there is little motivation for doing so. Higher order con. putations require symbol manipulation on a computer. The result is an analytic form for a higher order term, but so complicated as to have littie intuitive value. Remembering also that convergence is in doubt, and that only a few terms of time series can be handled, one is beller advised to seel: a nomperturbative numerical method.

One nou-perturbative approach is the simple surfaco-fitting method of the. previous section, which may well turn out to be the most practical techuiqur for getting a good first approximation in cases of strong nonlinearity. Anothe' useful approach is ilerative numerical solution of the Hamilton Jacobi muatiun. as outlined in Section 4. 


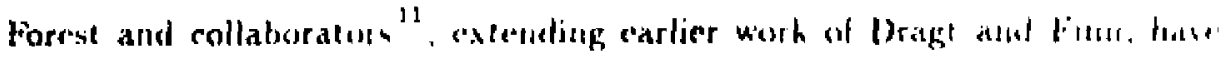
developed an improvenuevt to perturteation theory. which depends on prun tam struction of a full-turn map for propagation in the lattire. 'Ihe map is tratin

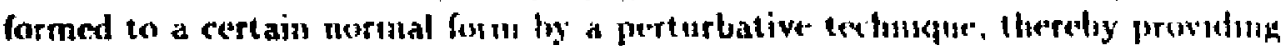
all analysis according to resunances, anct giving an approximate invariant surfar in implicit form. The advantage of this approach is that reperated intogratons over the lattice are avoided, making it feasjble for large lattices. The Hamiltorian and equations of motion are used only ouce, in construction of the map. The normal form analysis is still basically perturbative, however, and is probably mo more of fective than usual perturbation theory when nonlinearitics are strong Wo should reserve judgrnent on this puint, howev't, until nontrivial towlts with comparisons to tracking are publistied.

\section{HAMILTON-JACOBI METHOD.}

Percurbation theory is a method for approximate solution of the Hamiltou Jacobi equation (2.2), basinl on cxpansion in powers of the perturbation strength. There are other methods to find approximate solutions which are superior with respect to accuracy, region of convergence, and simplicily of programming. We have in mind iterative methons, ${ }^{8,12}$ which depend on writing the Hamilion-Jacoli equation as a fixed-point problem,

$$
g=A(g)
$$

Here $g=[9 \mathrm{~m}]$ is a vector made up of the Fourier coefficients of the generating function. The only approximation arises from truncation of the Fourier series. We have considered various formulations leading to different realizations of the nonlinear operator $A^{\mathrm{B}, 13,14}$ The best formulation to date for accelerator problems is that of Ref. 14 , in which the periodicity of $g_{\mathrm{m}}(s)$ in $s$ is achieved by a shooting method. In many cases the equation (4.1) can be solved by simple iteration,

$$
g^{(n+1)}=A\left(g^{(n)}\right)
$$

With $g^{(0)}=0$, the first iterate $g^{(J)}$ is identical with the result of lowest ordler perturbation theory; on the other hand $g^{(2)}$ entails all orders in lie perturloa tion strength. In difficult cases, for instance strongly nonlinear lattices near the dynamic aperture, the itrration (4.2) diverges unless the set of allowed modes is rather small. For such cases we can resort to the more puwerful Newton iteration and recover convergence even on relatively large mode sels. We refer the readut to Ref. 14 for details. 


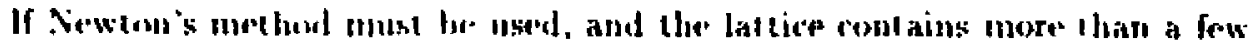

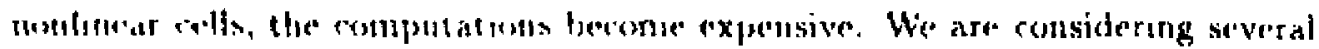

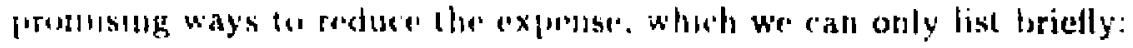

(1) Cse the nperuximate Kenerator. oblaimed ly a fit to tracking data as in

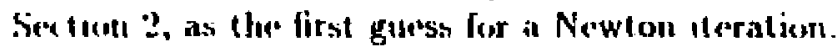

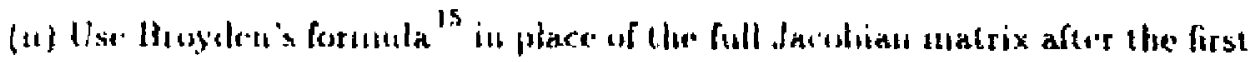
ilertate of Newlants method.

(iii) Ise plain iteration (t.2) wh a small moke set, perform a canotical trans formation with the tesultant gerlerator, and again do plain iteration on llio new Hamilion-Jatobi equation in the now variables, but now on a heger mede" set This is possible tocanas the muntuer of modes allowed in a convergent itcration increiases when the perturbation decreases in strenghth.

If the step of iten (iii) is repeated indefinitely, one has an arcelerated version of the K.- A.-M. algorithn, which lands to an invariant surface of arbitrary arcuracy. Although Newton's method as such is not used, the iteration will have super exponential convergence. In the past it has been awkward to carry out such a program, because of the nonlinetar rharacler of the equation (2.3) rolating old and new angle variables. In lief. 8 we introduced an effective nonperturbative method of solving (2.3), which is based on the Fourier expansion

$$
\phi=\psi+\sum_{m} A_{m}(J, s) e^{m} \psi
$$

It is casy to calculate the coefficients $A_{m}$ numerically, in terms of the generator $G$. We cepresent the J-dependence of $G$ by polynomial interpolation of solutions $G\left(J_{1}, b, s\right)$ computed at a fow values $J_{n}, i=1,2, \cdots, r$. The new Hamiltonian, $H^{(1)}(\mathrm{J}, \mathrm{T}, \mathrm{s})$, may be represented similarly as a Fourier series in $\Psi$.

The advent of a compact and managealile method for successive canonical transformations should open up new posibitities for precise results on stability. as explained in the following section.

For large lattices it may be advantageous to work with a lunctional equation for the invariant surface at a fxed value of 5 , in lieu of the Hamilton-Jacobi equation. Such an equation cas be formulated in terms of a full-turn map, as is indicated in Section 7. 


\section{CANONICAL TRANSFORMATIONS AND BOUNDS ON THE MOTION FOR TIME $T$}

Wh have meationed acveral ways to approximate invariant surfaces (tequiv. alently, periodic colutions $G$ of the Hanilton-Jacobi equation). Although the approximations that we obtain in practice seem rather close, in the sense that they agree with tracking to about I part in $10^{5}$ for several thousand turns, one bould asix about their real value in settling the stability question. Mathenatical andyeis (K.-A.-M. theory) assures the existence of exact invariant surfaces only on a strange eat of Cantor type in action space, and such surfaces cannot be rep. resented in terms of a finite number of Fourier modes. Also, the invariant surfaces do not divide phase space into disjoint regions if the number of degrees of freedom is sufficiently large ( $\geq 2$ for our cave of a periodic time-depcndent Hamiltonian, $\geq 3$ for autonomous systems). This allows Arnol'd Diffusion, a slow drift from cae region of phave epace to a far removed region. In view of this apparently discouraging theoretical situation, what is the meaning or value of an "approximate invariunt surface" ?

One answer is provided by the line of argument used in the Nekhoroshev Theorem. 17 An approximate invariant surface is associated with a generator of a canonical trantormation. Whatever the genesis or pedigree of this transformation. it has the property of reducing the perturbation term in the llamiltonian, so that the motion in the new variable is closer to being linear. For instance, in Rel. $8 \mathrm{tlut}$ transformation obtained from an approximate iterative solution of the HarriltonJacobi equation reduced the perturbation by a factor of $10^{7}$, even in a region not too far from the oneet of chaos. As in the final step of Nekhoroshev's argunueut (a trivial but important step) one can make a simple estimate using Hamilton's equations in the new variables. This estimate gives a lower bound on the time $T$ during which an orbit will stay in a chosen region of phase space. This rigorous bound allows for the complications mentioned above, Cantor sets and Arnol'd Diffusion.

The better the canonical transformation, i.e., the smaller the fesidual depen. dence of the transformed Harniltonian on $(4, s)$, the larger the lower bound on 7 '. This impels research in the direction of finding highly accuratc approximations to invariant surfaces, using if necessary the method of successive canonical transfor. mations as outlined in the previous section. We anticipate much better boutuls on $T$ then those of Nekhoroshev, since to prove a theorem he had to make and. lytical estimates, necessarily very crude, on a canonical transformation obtained from high-order perturbation theory. Since we compute our transformation III. merically, we have a closer knowledge of what it actually accomplishes. Moreover, there is no important lapse of rigor in resorting to numerical methods in this con. text, since the only source of error is in the problem of numerical determination 
of an upper bound for a well-defined function of a few variables. It is easy to show that the new action $J(t)$ will be confined to a strip

$$
|J(t)-J(0)|<\Delta J
$$

for $0<t<7$

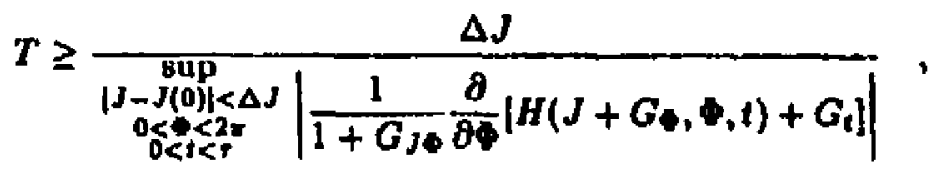

t being the time for one revolution. (We atate the formula for one degree of freedom; it is easily generalized). However $G$ was obtained, by fair means or foul, it is a precisely defined function given by a truncated Fouricr serich, and we should be able to evaluate $t_{i}$ " denominator of (5.2), or an upper bound to that denominator, without appreciable crror.

\section{FULL TURN MAPS}

Maps to describe propagation through one or more nonlinear elements of an accelerator or beam transport line have heen utilized for a long time, for instance in the code TRANSPORT since the early 1960's, The map

$$
\mathbf{M}\left(\mathbf{z} ; s, s^{\prime}\right)=\mathbf{z}^{\prime}
$$

takes a phase space point $\mathrm{z}$ at orbital location $s$ into a point $\mathrm{z}^{\prime}$ at orbital location $s^{\prime}$. It has been usual to rt'present $M$ as a power serjes in the components of z. Until tecently only the frst few Taylor coefficients could be computed in the case of a realistic full-turn map, even with the help of a Lie-algebraic formslism and symbol manipulation codes. By applying a technique lot antomatic differentiation ${ }^{19}$, which encodes the rules for differentiation of functions composed of truncated power series, one can go to much higher order. This technique is to be distinguished from derivative evaluation by symbol manipulation or by divided differences. It provides values that are exact to machine precision. Berz ${ }^{19}$ has implemented the method in codes which allow one to find the Taylor coefficients of the map defined by an urbitrary tracking program. In principle, derivatives of any order can be handled, but in practice there are restrictions due to bimited computer power. 
Although Berz's work certainly advances the art of Taylor strin's mipm. "We are not convinced that Taytor series provide the most eflicisut representation of the map M. Taylor series are rarely used in unodern numerical analysis for gluthal epproximation of functions. The reason is elementary: interpolat ion through mul tiple points is usually more accurate and more convenient than oxtrapolation by Taylor series from a single point. Orthogonal expansions, in particular Finsin series, also provide a tool with a murh wider range of validity than Taylur ex pansions. The practice and theory of interpolation and orthogonal expansions are" bighly developed ${ }^{20}$, and necessary softwate is rcadily availabls.

We have implemented a representation of maps using a combination of inter polation and Fourier developments. The data required to construct the maps are merely the values of $M\left(z ; s, s^{\prime}\right)$ on some set of initial $f$ sints $z=z_{1}$; the values can be taken from any tracking code by running it from $s$ to $s^{\prime}$ for each $z_{1}$. In contrant to Berz's method, we need not deal with the mathematical operations within the code. We write the map in terms of angle-action coordinates:

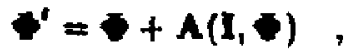

$$
\begin{aligned}
& I^{\prime}=I+B(1, \Phi)
\end{aligned}
$$

The functions $A$ and $B$ are periodic in $\$$ with period $2 \pi$. In view of general experience in nonlinear mechanics, it seems natural to expand these functions itt Fourier series in 4 . As in the computation of invariant surfaces, we find that a rather sparse set of Fourier modes is dominant. That is, for a given accuracy we have to include modes up to some maximum mode number, but for many mode numbers below that maximum the Fouricr coefficients are negligible. Thus, it is efficient to represent $\boldsymbol{A}$ and $B$ in $a$ form such ap

$$
A(\mathbf{l}, \boldsymbol{W})=\sum_{m \in S} A_{m}(\mathbf{I}) \mathbf{e}^{1 \mathrm{~m}} \cdot
$$

where the set $\boldsymbol{S}$ includes all $m$ for which $\left|\boldsymbol{A}_{\mathrm{m}}\right|$ is greater than some times the largeat $\left|A_{m}\right|$. The Fourier coefficients are calculated by evaluating the map on a rectangular grid of points in space, then taking an FFT of the values.

By aumerical evaluation we find that the Fourier coefficients of (6.4) are amooth, uneventful functions of $I$, usually monotonic and with small curvature over the region encountered on a typical orbit. We have experimented with two waye to repreant the functions: ${ }^{2}$ (a) interpolation by polynomials in the variables $G_{i}=l_{i}^{1 / 2}$, the $l i$ being components of the vector $I$, and (b) spline interpolation in the ame variables. Here we report results from choice ( $k$ ), while 
bening that rhenere (b) gives smilar resules itt must cases that we have tried. In onte dime-nsion, the polynomial approximation of the Fourier coethrient is given in l.algtange torm as

$$
A_{m}(J)=\sum_{s=1}^{n} A_{m}\left(l_{s}\right) \lambda_{s}(\xi)
$$

where the lagrange factors $\lambda_{9}(\xi)$ are

$$
\lambda_{s}(\xi)=\prod_{t \neq 1} \frac{\left(\xi-\xi_{t}\right)}{\left(\xi_{0}-\xi_{t}\right)}
$$

For the interpolation points $\xi$ we take the expanded Chebyshev points, as given in Eq. (2.Ge) of de Boor ${ }^{20}$, which in a certain sense make an optinal choice. The denominator of (6.6) is computed once for all, while the numerator is evaluated once for each iteration of the map.

To give an example we construct maps for the SLC North Damping Ring, to cover a region including the orbit that yielded Figures 1 and 2. The interpolation polynomials are sixth degree in each variable $\xi_{1}$ with minimum and maximum interpolation points at the ends of the intervals

$$
1.5 \cdot 10^{-6} \leq I_{1} \leq 3.25 \cdot 10^{-6}, \quad 10^{-6} \leq I_{2} \leq 2.5 \cdot 10^{-6},
$$

with actions given in meters. The Fourier modes are chosen Irom an initial sct with $\left|m_{1}\right|,\left|m_{2}\right| \leq 11$. For each of the four components of the map we retain all modes with coefficients larger than $10^{-1}$ of the largest coefficient, this selection being made at maximum values of the actions. For a one-turn map this yields a total of 223 coefficients to describe all four components of the map; for a two-turn map thete are 246. The initial, unselected set had 972 independent coefficients.

To test accuracy of the $\mathrm{s}_{\mathrm{n}}$-th iterate of the map, we compare its value to the corresponding value oblained from the underlying tracking code (a 4th-order symplectic integrator) that was used to construct the map. We define the discrepancy 6 between the map and the tracking code as

$$
\delta(n)=\frac{1}{4} \sum_{i=1}^{2}\left[\left|\frac{\Phi_{1}-\Phi_{i}^{i}}{\Phi_{i}}\right|+\left|\frac{I_{1}-I_{i}^{\prime}}{I_{1}}\right|\right]_{n-14 \text { torn }}
$$

The cootdinates from the tracking code have superscript t. those from the map do nat. 


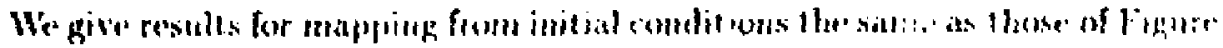

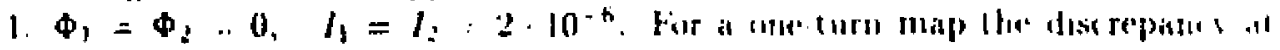

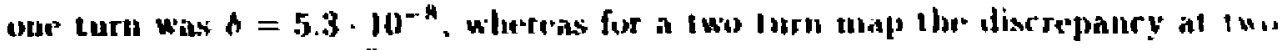
curns was $\delta=2.9 \cdot 10^{-8}$. In Tahle 2 we give llue djsirrepancoes for $10^{p}$ turns. wilh $p=1, \cdots, 4$. We find it remarkable that the discrepancy is quite smatl for al least 10000 turns. It is Jikely that the orbit gemerated by the map stays close to the correct invariant surface for many turns heyond 10000 , since it is usuat for phaseerror to build up faeter than amplitude crror. 'That is, the urbit germerated hy the map might lie close to the surface, without having the correct angular location $\left(\phi_{1}, \Phi_{2}\right)$ at a particular turn.

Table 2 : Discrepancy 6 between Map and Tracking Code

\begin{tabular}{|c|c|c|}
\hline$n=$ number of turns & $\delta(n)$ & $\delta(n)$ \\
& Oneturn map & Two-turn map \\
\hline 10 & $2.2 \cdot 10^{-7}$ & $6.8 \cdot 10^{-8}$ \\
100 & $1.6 \cdot 10^{-7}$ & $1.3 \cdot 10^{-6}$ \\
1000 & $79 \cdot 10^{-6}$ & $9.9 \cdot 10^{-6}$ \\
10000 & $5.8 \cdot 10^{-4}$ & $6.2 \cdot 10^{-4}$ \\
\hline
\end{tabular}

We have not imposed the symplectic condition, beyond maintaining good agreement with the underlying symplectic tracking code. It is possihle to en. force the symplectic condition precisely (modulo round-of error) by constructing a canonical transformalion that induces a map nearly the same as the one we have constructed. We have invented an algorithn, based on a Fourier inversion irhnique, to derive the generator of such a transformation from the map itself. It is also possible to fiud the generator by solving the Hanilton-Jacobi equation, later obtaining the explicit (but not exactly symplectic) map by the Fourier in. version method. This Jatter program, complementary and inverse to the present approach, was implemented in Ref. 23 in one degree of freedom.

Results concerning computation time to iterate the maps are encourngiug, especially in view of the fact that we have not yet done nuch to optimize con. puting. On the IBM 3081 at SLAC the time for one iteration of the one-turn map described ahove is about five times grealer than the time to track for one turn with the underlying tracking codr. Since the ring has 72 sextupoles, the map would go faster than element-by-element tracking in a ring with more than $5 \times 72=360$ sextupoles, provided that a map of the same complexity would suffice. In a ring as big as the SSC, with about 10000 nonlines elements, one would gain a factor of 26 


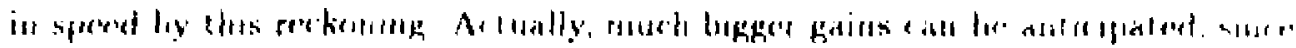

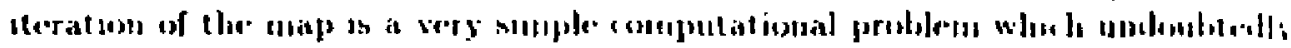

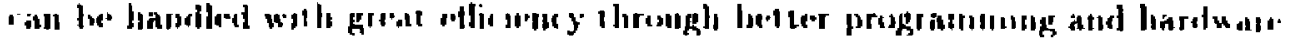

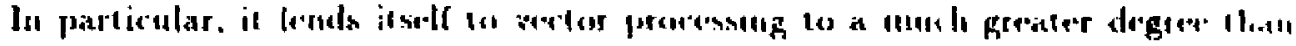
ordinary tracking.

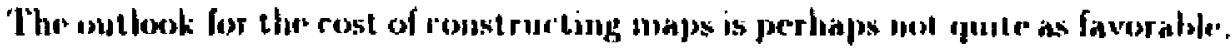
but by no means discouraging. It took the equivalent of 28224 turns of elenent by-clement tracking to construrt the one-turn map described above, and twice that much for the two-turn map. Furthernore, the maps do not cover the full domain of interest in action space. It Inight taku three times as much computiug to make maps for the full domain. Once the maps are available, however, they can be used to track economically (for large rings) from any initisl condition, and also to study invariant surfaces by the method of the following sertion. Furthermure, one could make naps for several values of tune, and interpolate between them to explore tune space.

It may very well be that the map of our example is much more arcurate than neressary. If the symplartic condition were enforced, a less accurate and less expeusive map might suffire. In order to check accuracy while allowing for innocuous phase error, one could calculate the distance between the orbit of a proposed map and an accurate, previously established invariant surface. That is, if the map gave the point $(1, \Phi)$ at sotne iterate, one could compare I with the action value on the invariant surface at tho same $\Phi$.

Tine question of whether one should work with a one-turn map or a multi-turn map deserves continued study. In Table 2 we see that the one- and two-turn maps give comparable accuracy at the $n$-th turn. Tracking by the two-turn map goes almost twice as fast (not exactly twice as fast, because it has a few more Fouricr modes) but the map takes twire as long to construct. Using the two-turn map. one night be ahle to study intariant curves in the neighborhood of a closed orbit of period two, by the method of the following section. Also, one could imagine a bootstrap operation in which a one-turn map, say, could be used to generate a map for many turns. Essentially this is what we have already done in going from the single-elenent maps of the tracking cove to full-turi maps.

Talman's ${ }^{21}$ recent study of maps for a simple model (the physical pendulum) led to a nore pessimistic outkok than wr have attained, but gave one result with which we agrex: maps based on interpolation are more promising than those based on the Taylor expansion. Hagel and Zutter, ${ }^{22}$ using slightly more relevant but still very simple models, have reexamined Tulnuan's conclusions. We have demonstrated that one ran easily make maps for real arcelerators, using any tracking code; it is not necessary, and potentially misleading, to work with over-simplified models. 


\section{FUNCTIONAL EQUATION FOR INVARIANT SURFACF.S}

In his original paper on the K.-A.-M. theorem, Jürgen Moser ${ }^{24}$ st mulerl 1 lı" invariant curves of area preserving maps of an annulus. He based his analys alu a functional equation for the' curve. We seck to generalize Mose'r's approach. sw as to capture its advantages in the case of a general Hamilionian systrm.

We begin with an $n$-turn map in the form $(6.2),(6.3)$, where" $n$ is an intower. say $n=1$. The map may be represented explicitly, as in the work of the previ. ous section, or may be regarded as the result of applying an cleme'nt by colvinent tracking code for $n$ turns. The desired invariant surface may he paramet rized in terms of the angular torus coordinates and developed in a Fourier serir's, just as in (2.1). Replacing the notation of (2.1) we write

$$
I=u(\varphi)=\sum_{m} u_{m} e^{i m \cdot \bullet}
$$

where $u_{v}$ is the invariant action $K$. The requirement that the surface be invariant under the map is

$$
\mathbf{I}=\mathbf{u}(\boldsymbol{\zeta}) \quad \Rightarrow \quad \mathbf{r}^{\prime}=\mathbf{u}\left(\boldsymbol{s}^{\prime}\right)
$$

In terms of the functions $A, B$ of $(6.2),(6.3)$ this means that

$$
\mathbf{I}+\mathbf{B}(\mathbf{I}, \mathbf{S})=\mathbf{u}(\mathbf{T}+\mathbf{A}(\mathbf{I}, \mathbf{T}))
$$

whenever $1=u(\$)$, which is to say

$$
\mathbf{u}(\boldsymbol{\varphi})=\mathbf{u}(\boldsymbol{\varphi}+\mathbf{A}(\mathbf{u}(\boldsymbol{\varphi}), \boldsymbol{\varphi}))-\mathbf{B}(\mathbf{u}(\boldsymbol{\varphi}), \boldsymbol{\Phi})
$$

Eq. (7.4) is the generalized Moser equation. (Actually, Moser used an auxiliary curve paramiter rather than the torus coordinate $\$$ to parametrize his invariant curve, which led to a pair of equations rather than one).

If we introduce (7.1) in (7.4), then take the Fourier transform of $(7,1)$. wor obtain a nonlinear system of equations for the coefficients $u_{2 z}$. After trums al ion of the Fourier ser: $\mathrm{m}$, the system can be solved by iteration, as in our treatment of the Hamilton-Jacobi equation. There are several possible iteration schemes, includilig - scheme similar to Moser's method of successive (non-canonical!) coordiuate. changes, which should allow one to include more and more Fourier modes as the iteration goes on, and thus achieve very high accuracy. 
Alt hough we have not yet implernented this method, it seems highly promising in light of our experience with the Hamilton-Jacobi system, which has smilal' mathemat ical propertiess, but in mure awk ward to handle in a context of successive roordionte rhanges. The method yields $C_{\phi}$ at one point in the lattice, say $s=0$. which can be taken as the initial value in an integration of the Hamilton- Jacolsi equation with respect to s over the whole lattice, $t u s=C$. 'Thus we obtain thegentrator for a canonical transformation whiclu can lead to bounds for long tet In! stability, along the lines of Section 5.

As was remarked in Seclion 3, this appronch retains the advantage of forest 's stheme by working with the map rather than the Hamiltomian, but allows precise. non-perturbative solutions.

\section{CONCLUSION}

We have discussed two methods for single-particle tracking: synplectic integration and full-turn maps. These techniques support and complenent three melhods for determination of invariant surfaces: surface fitting, the HasmiltonJacobi method, and the generalized Moser method. Knowledgr: of approximate in. variant surfaces is essentially equivalent to knowledge of an approxinate llamilouJacobi generator, which leads to precise bounds on the motion over a finite time $T$. It is too carly to say whether $T$ will be large enough to be interesting; we have mentioned the possibility mainly to point out a long range goal, the ideal completion of the program through definite statements on long term stability.

Numerical work to date has been devoted to assessing the relative advantages of the various approaches. This aspect of the task is still not complete, but results are sufficient to convince us that real advances in the art are possible. As soon as a preferred method comes into focus, we hope to lake advantage of advanced programming and hardware, and also produce software for general use.

Finally, we should mention that the methods under st udy should be of interest beyond accelerator theory, for instance in chemical physics (througl semi-classical quantum mechanics) and in celestial mechanics. 


\section{REFERENCES}

1. R. De Vogelaere, Dept. of Math., University of Notre Dame, Roport 4, 1!50.

2. R.D. Ruth, IEEE Trans. Nucl. Sci., 30 (1983) 2669.

3. P. Channel!, LANL Tech. Note AT-6:A'N-83-9, Los Alamos National Laboratory, 1983

4. P.J. Channell and C. Scovel, LA-Uli-88-1828, Los Alaynos National Laho. ratory, 1988, submitted to Nonlinearity.

5. R.D. Ruth, unpublished. The 4 th-order program TRACli was written at SLAC by Karl Ecklund.

6. Notes on the Los Alamos Workshop on Symplectic Integration, Marcli 19-21, 1988, Daryl Holm, Ed.

7. D. Edwards, SSC-2 (1985); T. Collins, SSC-26 (1985); Proceedings of the Second Aduanced ICFA Beam Dynamics Workshop, CERN 88-04 (1988), working group summaries by S. Chattopadhyay and P. Alldy et al.

8. R.L. Warnock and R.D. Ruth, Physica 26D,1 (1987).

9. L. Michelotti, Particle Accelerators 16 (1985) 233.

10. H. Moshammer and J. Hagel, Proceedings of the Second Aduanced ICFA Beam Dynamics Workshop, Lugano, April 11-16, 1988, CERN 88-04.

11. E. Foreat and M. Berz, LBL-25069 (1988); E. Forest, M. Berz, and J. Irwin, SSC-166 (1988); E. Foreat, Particle Accelerators 22,15 (1987), SSC-29 (1985); A.J. Dragt and E. Forest, J. Math. Phys. 24, 2734 (1983).

12. S. Chapman, B. C. Gartett and W. H. Miller, J. Chem. Phys. 64 (1976) 502.

13. R.L. Warnock and R.D. Ruth, Proceedings of the 1987 IEEE Particle Accelerator Conference, p.1263.

14. W.E. Gabella, R.D. Ruth, and R.L. Warnock, Second Aduanced ICFA Beam Dymamics Workshop, Lugano, 1988, CERN 88-04.

15. C. G. Broyden, Math. Comp. 19 (1965) 577.

16. R.L. Warnock and R.D. Ruth, in The Physics of Phase Space, Lecture Notes in Physics, No.278, Springer-Verlag, 1987.

17. N. N. Nekhoroshev, Russian Math. Surveys \$2:6 (1977) 1.

18. K.L. Brown, D.C. Carey, Ch. Inelin, and F. Rothacker, TRANSPCRT, a Computer Prognam for Designing Charged Parlicle Beam Transport Systemo, CERN 80-04, 1980; K.L. Brown, R. Belbeoch, and P. Bounin, Rev. Sei. Instr. 95 (1904) 481. 
19. M. Berz, SSC-166, March, 1986. For earilier work on antentitic differentiation in the same vein see $R$. F. Wengert, Commun, of the $\Lambda \mathrm{CM}, 8$ \{1!6i-1) 463; L.H. Rull, Automatic Diferentiation: Techniques and Applacntions, Lecture Noles in Conputer Srience, Vol.120, Springer, Berlin, 1981; H. Kagiwada, It. Kalaba, N. Rasitkhoo, and K. Spingarn, Numerical Derivatives and Nonlinear Analysis, Plenum, New York, 1986. See also the review of the latler book by A. Griewank, SIAM Jleview, 50 (1988) 327 Griewank's review contains references not mentioned in the two monographs.

20. C. de Boor, A Practical Guide to Splines, Springer, Berlin, 1978; T. Rivlin, Approrimation of Functions.

21. R.Talman, SSC-117, 1988.

22. J. Hagel and B. Zotter, CERN-LEP report, 1988.

23. R. L. Warnock, R. Ruth, and W. Gabella, Symplectic Maps for Accelcrator Lattices, Workshop on Symplectic Integration, Los Alamos National Laboratory, March, 1988, SLAC-PUB 4627.

24. J. Moser, Nachr. Akad. Wiss., Göttingen, Math. Phys. Kl., 1-20, 1962. 